\title{
Investigation on Magnetic Properties of Soft/Hard Magnetic Bilayers at Different Temperatures: a Monte-Carlo Study
}

\author{
C. DeĞER ${ }^{a, *}$, M.E. AKÖZ ${ }^{b, c}$, M. ERKOVAN ${ }^{b, d}$ AND F. YILDIZ ${ }^{b}$ \\ ${ }^{a}$ Marmara University, Dept. of Physics, 34722, Göztepe, Istanbul, Turkey \\ ${ }^{b}$ Gebze Technical University, Dept. of Physics, 41400, Çayırova, Kocaeli, Turkey \\ ${ }^{c}$ Yildiz Technical University, Dept. of Physics, 34220, Esenler, Istanbul, Turkey \\ ${ }^{d}$ Sakarya University, Dept. of Nanoscience and Nanoengineering, 54187, Serdivan, Sakarya, Turkey

\begin{abstract}
Soft/hard magnetic bilayers are attractive systems for investigating their physical behavior due to scientific curiosity and the potential for technological applications. Thus, $10 \mathrm{~nm}$ thick hard magnetic layer placed on a $20 \mathrm{~nm}$ soft magnetic layer was modeled in this work. After the constructing of magnetic thin films, the bilayer structure was simulated based on micro-magnetic modeling. When constructing the Hamiltonian of the system, magnetocrystalline anisotropy term was also under debate with classical Zeeman and exchange terms. Energy minimization in the simulation was performed using Metropolis algorithm and Markov Chain Monte Carlo (MC-MC) method. As a result of modeling, hysteresis curves, remanent magnetization and coercive fields were obtained at different temperatures.
\end{abstract}

DOI: 10.12693/APhysPolA.129.869

PACS/topics: $75.50 . \mathrm{Cc}, 21.60 . \mathrm{Ka}$

\section{Introduction}

Magnetic multilayers are currently attracting a great interest because of their expected application in spintronic devices [1]. For spintronic applications, magnetic multilayers could be used in magnetic field sensors, magnetic read heads, and magnetic random access memories [2]. Magnetic properties of materials used in technological applications must be completely known at different conditions. So, experimental and theoretical investigations of material properties were frequently considered in previous studies [3, 4]. Experimental results can be drastically changed under different conditions. Especially the temperature has a crucial role in magnetic properties. Because of that, our aim was set to a next generation of scientific studies. On this way, we have used one of the popular methods in recent years, the Monte Carlo method.

Monte Carlo methods are very useful computational algorithms that rely on repeated random sampling to obtain numerical results for systems with many coupled degrees of freedom, such as disordered materials, cellular structures and strongly coupled solids [5]. Magnetic multilayer structure studied by us, has two different ferromagnetic materials, a soft magnetic and a hard magnetic material. Ferromagnetic materials may be either soft or hard depending on the magnetic character of materials [6]. We have tried to simulate hard magnetic bilayer on top of a soft magnetic bilayer to observe their magnetic

\footnotetext{
*corresponding author; e-mail: caner.deger@marmara.edu.tr
}

behavior. We have investigated $\mathrm{Fe}_{14} \mathrm{Nd}_{2} \mathrm{~B}(10 \mathrm{~nm})$ as hard magnetic material on top of $\alpha$-Fe $(20 \mathrm{~nm})$ as soft magnetic material (Fig. 1).

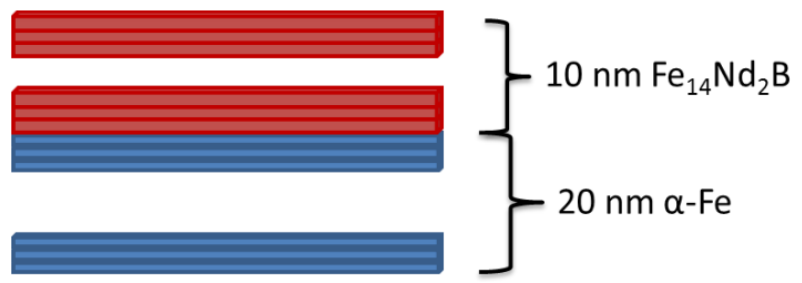

Fig. 1. Schematic view of $\alpha-\mathrm{Fe} / \mathrm{Fe}_{14} \mathrm{Nd}_{2} \mathrm{~B}$ bilayer structure.

Magnetization is a characteristic property of all magnetic materials and can be measured to reveal magnetic behavior, especially for ferromagnetic and ferrimagnetic materials. Based on hysteresis loops (magnetization curves), we can have information about remanence/saturation of magnetization and coercive field at different temperatures.

In this study, we have observed that magnetic multilayer structure at different temperatures ranging from $0.014 k_{\mathrm{B}} T / \mathrm{J}$ to $0.019 k_{\mathrm{B}} T / \mathrm{J}$. Markov Chain Monte Carlo method (MCMC) was used to analyze aforementioned magnetic system. Construction of bilayer structure, including randomly oriented sublayers was followed by evaluation of the orientation of sublayers as a function of external magnetic field and temperature.

\section{Theoretical model}

Monte Carlo Metropolis algorithm is an excellent way to study a physical system under equilibrium conditions. 
Making an adjustment to a physical system and calculating the change in energy, $\Delta E$, is the basic idea of Metropolis algorithm. This change is then accepted or rejected in relation to the Boltzmann distribution for the system at a given temperature [7]. In the case of a classical spin model a single spin is speculatively moved in space and the difference in energy is evaluated. The move is then accepted with probability $P$ according to

$$
P=\max \left(1, \exp \left[-\frac{\Delta E}{k_{\mathrm{B}} T}\right]\right),
$$

where $k_{\mathrm{B}}$ is the Boltzmann constant, and $T$ is the absolute temperature [7].

We have performed a micromagnetic calculation of the magnetization curves for $\alpha$-Fe $/ \mathrm{Fe}_{14} \mathrm{Nd}_{2} \mathrm{~B}$ multilayers using Landau-Lifshitz-Gilbert equation [8]. First, $\alpha$ $\mathrm{Fe} / \mathrm{Fe}_{14} \mathrm{Nd}_{2} \mathrm{~B}$ bilayer structure was divided into $2000 \mathrm{mi}-$ cromagnetic layers inside $20 \mathrm{~nm}$ of $\alpha$-Fe and 1000 micromagnetic layer inside $10 \mathrm{~nm}$ of $\mathrm{Fe}_{14} \mathrm{Nd}_{2} \mathrm{~B}$. The Hamiltonian has taken the form as:

$$
H=E_{\text {zeeman }}+E_{\text {exchange }}+E_{\text {mag. ani. }} .
$$

The magnitude of anisotropy constant for $\mathrm{Fe}_{14} \mathrm{Nd}_{2} \mathrm{~B}$ was taken to be $4.3 \times 10^{6} \mathrm{~J} / \mathrm{m}^{3}$ and that for $\alpha$-Fe was $4.6 \times 10^{6} \mathrm{~J} / \mathrm{m}^{3}[9]$. Three kinds of exchange interactions between magnetic layers have been considered of which two intra-layer coupling constants $J_{\text {Hard }}$ (between layers of $\mathrm{Fe}_{14} \mathrm{Nd}_{2} \mathrm{~B}$ ) and $J_{\text {Soft }}$ (between layers of $\alpha$-Fe) are fixed to be $2 \times 10^{-2} \mathrm{~J} / \mathrm{m}^{2}$, which is comparable to the values adopted by Fukunaga et al. [3, 10]. The interlayer coupling constant was taken to be $2 \times 10^{-3} \mathrm{~J} / \mathrm{m}^{2}$.

\section{Results and discussion}

$M(T)$ curve has been calculated via Markov Chain Monte Carlo method in the temperature range 0-0.04 $k_{\mathrm{B}} T / \mathrm{J}$ (Fig. 2). The sharp reduction of $M$ between 0.016 and $0.024 k_{\mathrm{B}} T / \mathrm{J}$ suggests that Curie Temperature $\left(T_{\mathrm{C}}\right)$ of our sample is nearly $0.02 k_{\mathrm{B}} T / \mathrm{J}$. It is clearly seen that thermal agitations cause random alignment of micromagnetic sublayers after $0.02 k_{\mathrm{B}} T / \mathrm{J}$. Above this temperature, the high value of $k_{\mathrm{B}} T / \mathrm{J}$ ratio forces system to paramagnetic behaviour. During the FC (Field Cooling) measurement, $4 \mathrm{kA} / \mathrm{m}$ external magnetic field was applied.

Magnetization hysteresis curves for certain temperatures are shown in Fig. 3. Remanent magnetization and coercive field values were determined for temperature range between 0.014 and $0.019 k_{\mathrm{B}} T / \mathrm{J}$. All data obtained from the simulation are given in Table I.

Table I shows that with the decreasing temperature, the magnetization values that indicate ferromagnetism are increasing. We have found that for $\alpha-\mathrm{Fe} / \mathrm{Fe}_{14} \mathrm{Nd}_{2} \mathrm{~B}$ bilayer structure, the temperature dependence of magnetization is roughly in accordance with the estimation based upon the simple superposition of magnetization of the $\alpha$-Fe and $\mathrm{Fe}_{14} \mathrm{Nd}_{2} \mathrm{~B}$ phases $[11,12]$.

Table I shows that remanent magnetization decreases to nearly zero at $0.019 k_{\mathrm{B}} T / \mathrm{J}$ indicating the existence of transition from a magnetically ordered state,

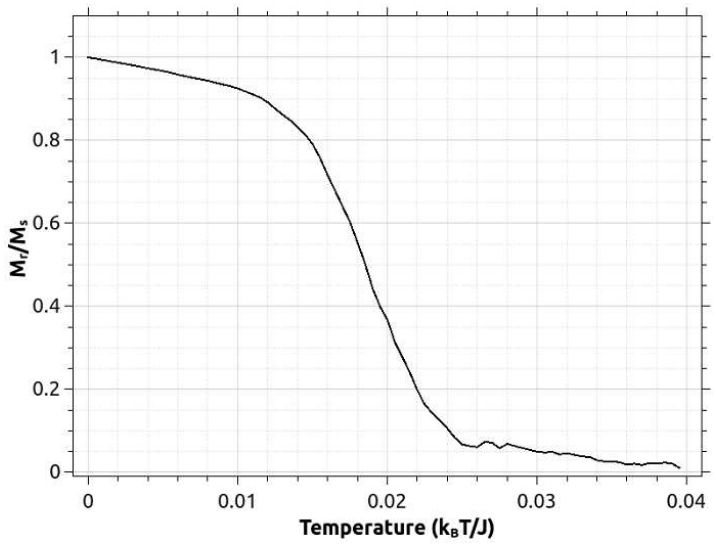

Fig. 2. The change of remanent magnetization as a function of temperature between 0 and $0.04 k_{\mathrm{B}} T / \mathrm{J}$.

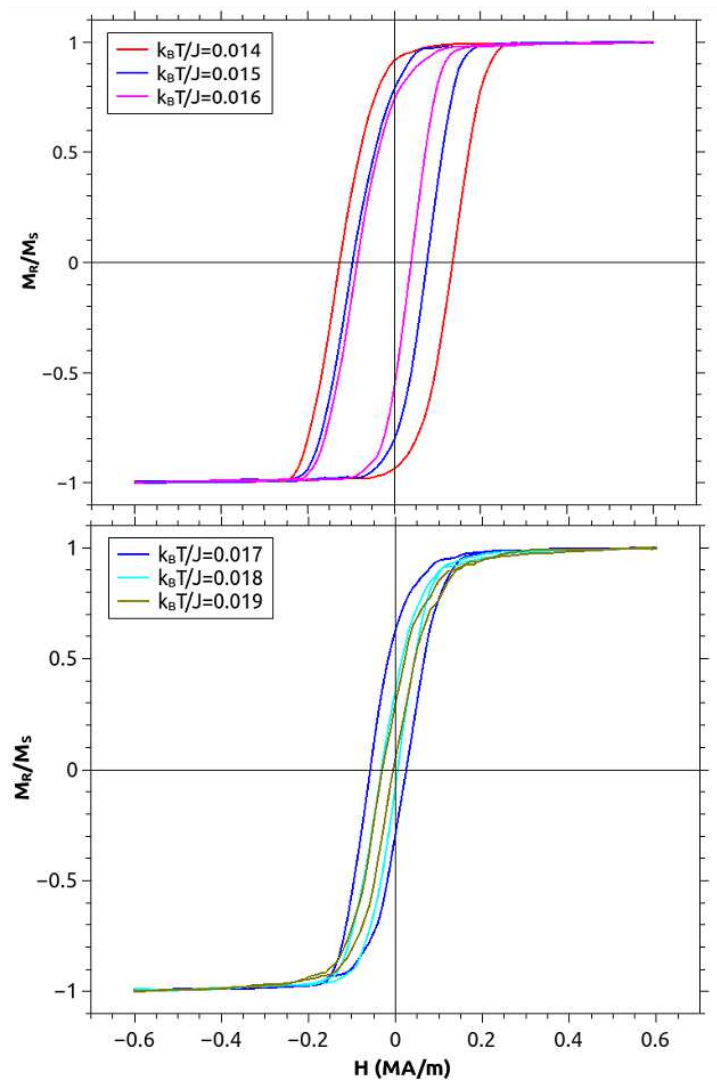

Fig. 3. Magnetization curves for $\alpha-\mathrm{Fe} / \mathrm{Fe}_{14} \mathrm{Nd}_{2} \mathrm{~B}$ bilayer structure at different temperatures.

ferromagnetic, to a magnetically disordered state, paramagnetic. The variation of MCMC method parameters with different temperatures can be considered. An exponential decrease of coercive field indicates that thermal agitations become dominant when compared to exchange coupling interaction with increasing temperature. The hysteresis curves of $\alpha$-Fe $/ \mathrm{Fe}_{14} \mathrm{Nd}_{2} \mathrm{~B}$ at different temperatures are compared in Fig. 3. The hysteresis curve gradually flattens as the temperature approaches the Curie point $\left(k_{\mathrm{B}} T / \mathrm{J}=0.020\right)$. 
TABLE I

The deduced magnetic parameters for all considered temperatures.

\begin{tabular}{c|c|c|c|c}
\hline \hline $\begin{array}{c}T \\
{\left[k_{\mathrm{B}} T / \mathrm{J}\right]}\end{array}$ & $\begin{array}{c}H_{\mathrm{C} 1} \\
{[\mathrm{MA} / \mathrm{m}]}\end{array}$ & $\begin{array}{c}H_{\mathrm{C} 2} \\
{[\mathrm{MA} / \mathrm{m}]}\end{array}$ & $\begin{array}{c}H_{\mathrm{C}} \\
{[\mathrm{MA} / \mathrm{m}]}\end{array}$ & $M_{\mathrm{R}} / M_{\mathrm{S}}$ \\
\hline 0.014 & 0.1367 & 0.1250 & 0.1308 & 0.9138 \\
0.015 & 0.0769 & 0.0951 & 0.0860 & 0.7834 \\
0.016 & 0.0395 & 0.0843 & 0.0619 & 0.7366 \\
0.017 & 0.0260 & 0.0573 & 0.0416 & 0.6273 \\
0.018 & 0.0070 & 0.0317 & 0.0193 & 0.3544 \\
0.019 & -0.0045 & 0.0293 & 0.0124 & 0.2992
\end{tabular}

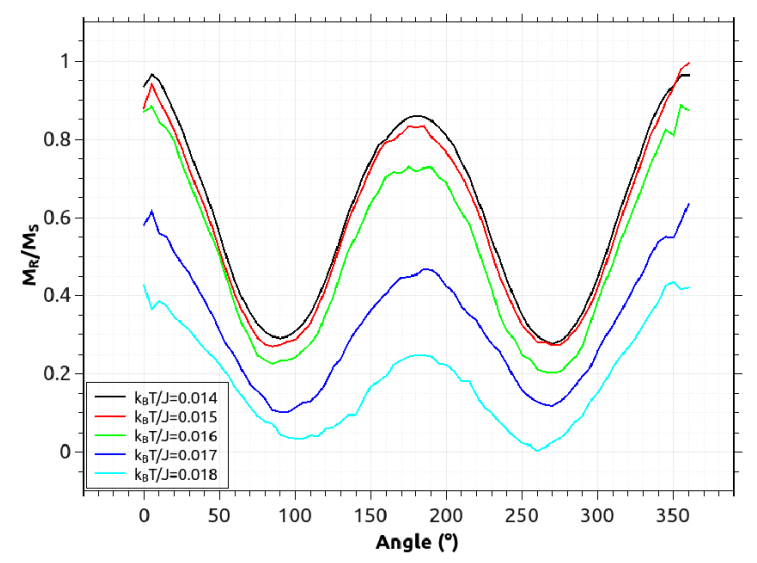

Fig. 4. In-plane magnetization data for angular dependence of $M_{\mathrm{R}} / M_{\mathrm{S}}$ from $\alpha-\mathrm{Fe} / \mathrm{Fe}_{14} \mathrm{Nd}_{2} \mathrm{~B}$ bilayer structure at temperatures between 0.014 and $0.018 k_{\mathrm{B}} T / \mathrm{J}$.

Angular dependence of magnetization of the sample at different temperatures is given in Fig. 4. In this figure all curves represent data obtained while the thin film was rotated from $0^{\circ}$ to $360^{\circ}$. Magnetocrystalline anisotropy energy in the Hamiltonian of theoretical model causes uni-axial two-fold magnetic anisotropy. There is no significant change in the behavior of magnetic anisotropy with increasing temperature while remanent magnetization of the sample is gradually decreases for all considered angles.

\section{Conclusions}

The multilayer magnetic thin films consisting of different ferromagnetic materials, of hard magnetic $\mathrm{Fe}_{14} \mathrm{Nd}_{2} \mathrm{~B}$ and soft magnetic $\alpha$-Fe have been investigated by Monte Carlo Metropolis algorithm. The characteristic magnetic parameters have been deduced using the theoretical model. Curie temperature $\left(T_{\mathrm{C}}\right)$ of bilayer system was determined as $0.02 k_{\mathrm{B}} T / \mathrm{J}$. Coercive field of sample was decreasing from $0.130 \mathrm{MA} / \mathrm{m}$ to $0.012 \mathrm{MA} / \mathrm{m}$ with temperature increasing from $k_{\mathrm{B}} T / \mathrm{J}=0.014$ to $k_{\mathrm{B}} T / \mathrm{J}=0.019$. Also $M_{\mathrm{R}} / M_{\mathrm{S}}$ ratio was decreasing from 0.913 to 0.299 for the easy magnetization axis of the bilayer structure. Two-fold in-plane magnetic anisotropy was observed for $10 \mathrm{~nm}$ of hard magnetic $\mathrm{Fe}_{14} \mathrm{Nd}_{2} \mathrm{~B}$ on top of $20 \mathrm{~nm}$ of soft magnetic $\alpha$-Fe at all investigated temperatures. There was no change in anisotropic behavior of bilayer structure with changing temperature. Synthesis and experimental analysis of soft/hard magnetic bilayer structures are planned as a future work.

\section{Acknowledgments}

The authors wish to acknowledge to Prof. Dr. Şahin Aktaş and Izzet Paruğ Duru for their technical support. This work was supported by Marmara UniversityBAPKO for the project FEN-C DRP-120613-0273.

\section{References}

[1] R.S. Hassan, C. Mény, N. Viart, C. Ulhaq, G. Versini, J.L. Loison, G. Pourroy, New J. Phys. 9, 364 (2007).

[2] C.H. Tsang, R.E. Fontana Jr., T. Lin, D.E. Heim, B.A. Gurney, M.L. Williams, IBM J. Res. Development 42, 103 (1998).

[3] M. Shindo, M. Ishizone, A. Sakuma, H. Kato, T. Miyazaki, J. Appl. Phys. 81, 4444 (1997).

[4] R. Topkaya, M. Erkovan, A. Öztürk, O. Öztürk, B. Aktas, M. Özdemir, J. Appl. Phys. 108, 023910 (2010).

[5] B. Rai, Molecular modeling for the design of novel performance chemicals and materials, CRC Press, 2012.

[6] B.D. Cullity, C.D. Graham, Introduction to magnetic materials, John Wiley \& Sons, 2011.

[7] R.F.L. Evans, Ph.D. Thesis, The University of York, 2008.

[8] H.N. Bertram, J.-G. Zhu, Solid State Phys. 46, 271 (1992).

[9] T. Leineweber, H. Kronmüller, J. Magn. Magn. Mater. 176, 145 (1997).

[10] J. Kuma, N. Kitajima, Y. Kanai, H. Fukunaga, J. Appl. Phys. 83, 6623 (1998).

[11] H. Jiang, M. O'Shea, J. Appl. Phys. 87, 6131 (2000).

[12] W. Dürr, M. Taborelli, O. Paul, R. Germar, W. Gudat, D. Pescia, M. Landolt, Phys. Rev. Lett. 62, 206 (1989). 